EXTENDED REPORT

\title{
Dose escalation of infliximab in clinical practice: improvements seen may be explained by a regression-like effect
}

\author{
R F van Vollenhoven, S Brannemark, L Klareskog
}

See end of article for authors' affiliations

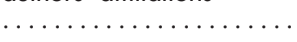

Correspondence to: Dr Ronald F van

Vollenhoven, Department of Rheumatology, Karolinska Hospital, D2-1, 17176 Stockholm, Sweden; ronald. vanvollenhoven@ks.se

Accepted 29 June 2003 Published online first 27 February 2004

\begin{abstract}
Objective: To determine whether increased infliximab doses result in better clinical outcome in rheumatic diseases.

Methods: Subjects were 124 patients with rheumatoid arthritis treated with biological agents at a single institute. Index cases were 44 patients whose infliximab doses had been increased. Controls were patients treated with infliximab without dose increase $(n=44)$, and patients treated with etanercept $(n=36)$. Disease activity score (DAS28), ACR28 swollen joint counts, and numerical ACR responses were compared before and after dose increases. For the controls, the point at which the DAS28 value showed any increase (despite infliximab/etanercept treatment) was used as the reference time point. Comparisons were made between three sets of outcomes: best outcome achieved before the dose increase (cases) or before the reference time point (controls); outcomes at this point; and best outcomes after this point.

Results: Following dose increase, disease activity showed modest but statistically significant improvements. The improvement achieved after dosage escalation was equal to, but not better than, the best values before dose escalation. While this finding could be interpreted as "recapturing" the previous response, similar improvements were seen in both control groups. Thus the same pattern of worsening and subsequent improvement was seen with or without the infliximab dose increase.

Conclusions: Clinical improvement with increased infliximab dose, and the impression that a previous response can be "recaptured" with higher doses, cannot be taken at face value, as similar improvements occurred in two control groups. The use of infliximab at doses higher than $3 \mathrm{mg} / \mathrm{kg}$ needs to be evaluated further.
\end{abstract}

nfliximab has been approved as treatment for rheumatoid arthritis in many countries. After three initial infusions within six weeks, the usual maintenance treatment is one infusion every eight weeks. While the normal dose is $3 \mathrm{mg} / \mathrm{kg}$ for each infusion, a dose of $3-10 \mathrm{mg} / \mathrm{kg}$ body weight per infusion is given in the US package insert, and doses in this range are widely used in clinical practice. The dose range is based on published data from the ATTRACT clinical trial (anti-tumor necrosis factor trial in rheumatoid arthritis with concomitant therapy), where some outcomes appeared to show a dose-response relation. However, the prospectively determined primary outcome did not differ significantly by treatment dosage. ${ }^{1}$ No clear guidance regarding dosage escalation has emerged from clinical trials or from other sources of longitudinal follow up data. While the overall safety profile of infliximab has not shown major dose related toxicity, at least one specific concern has been raised about the possibility of an increased risk with higher doses-in a small study of patients with severe congestive heart failure there appeared to be an increase in overall mortality and a worsening of cardiovascular function, particularly in the patients given ínfusions at $10 \mathrm{mg} / \mathrm{kg} .{ }^{2}$ In addition, the use of higher infliximab doses is associated with significantly increased costs.

In communicating with rheumatologists nationally and internationally, it has become clear that infliximab dose escalation is used very often. The two reasons given for this are inadequate results with the original dose, and a treatment effect that lasts for less than the planned interval between infusions.

While controlled clinical trials may provide the most reliable answer to specific questions regarding single com- parisons, the more complex questions that govern clinical decision making can rarely be addressed adequately using randomised trials. ${ }^{3}$ In such instances, structured longitudinal follow up studies may have greater practical value.

We now have at our disposal such a structured follow up system for patients being treated with biological agents in the rheumatic diseases, namely the STURE registry. We used data from this registry to address the question of whether increasing the dose of infliximab above $3 \mathrm{mg} / \mathrm{kg} /$ infusion results in clinical benefits sufficient to outweigh the potential increase in risks and the definite increase in costs.

\section{METHODS}

The STURE database (Stockholm TNF $\alpha$ follow up registry) collects efficacy and safety data for all patients starting biological treatments at the major hospitals in Stockholm, as part of the nationwide registry of antirheumatic therapies in Sweden (ARTIS). The assessments are done at 0, 3, 6, and 12 months and annually thereafter, and include the American College of Rheumatology (ACR) core outcomes (the 28 swollen and tender joint count, visual analogue scales for global health and pain, the health assessment questionnaire disability index (HAQ), erythrocyte sedimentation rate,

\footnotetext{
Abbreviations: ACR, American College of Rheumatology; ACR-N, numeric ACR joint response; ARTIS, antirheumatic therapies in Sweden database; ATTRACT, anti-tumor necrosis factor trial in rheumatoid arthritis with concomitant therapy; DAS28, 28 joint count based disease activity score; EULAR, European League Against Rheumatism; STURE, Stockholm TNF $\alpha$ follow up registry; TNF, tumour necrosis factor
} 
C reactive protein, and physician's global assessment of disease activity); the 28 joint count based disease activity score $(\text { DAS28 })^{45}$; records of concurrent drug treatments; employment status; and side effects. In the study reported here, we analysed data on those patients whose infliximab dose was increased to $5-7 \mathrm{mg} / \mathrm{kg}$ per infusion. At our institution, during the period of this study, all infusions were given at eight week intervals. Based on these data, the following comparisons were made:

- Comparison of disease activity by DAS28, swollen joint count, and ACR-N (numeric ACR) response ${ }^{6}$ at the evaluation before a dose increase (that is, at the time when the decision to increase the dose was made) with the values after the dose increase. The dose increases were implemented at the next scheduled infusion, at a median of three weeks after the decision was made, and the first assessment thereafter would occur according to the schedule of visits for the registry.

- Comparison of the best DAS28, swollen joint count, and ACR-N response seen in these patients after the dose increase, and comparison of these values with each patient's best result at any time point before the dose increase. These clinical responses were also analysed using established EULAR (European league against rheumatism $)^{7}$ and $\mathrm{ACR}^{8}$ response criteria.

- Comparison with two control groups, one formed of patients who were treated with infliximab but without dose increase and one of patients who were treated with etanercept (dose increases of etanercept are not employed at our centre). Analysis of the control groups was based on the hypothesis that infliximab dose increases are "triggered" by deteriorating clinical results. Therefore in control patients the analysis of clinical results was anchored at the time of the first increase in DAS28 after treatment started (the reference time point). We then made the following comparisons: first, between the best values for DAS28, swollen joint count, and ACR-N at any time before the dose increase or the reference time point; second, between the values at the time of dose increase or the reference time point; and finally, of the best values after the dose increase or the reference time point.

While the STURE database is part of the ARTIS national biologicals safety registry, this study involved only data collected and analysed at the Karolinska Hospital.

Statistical analyses were done using StatView 5.0.1 for PC (SAS Institute Inc, Cary, North Carolina, USA).

\section{RESULTS}

\section{Disease activity after infliximab dose increase}

We evaluated 44 patients whose infliximab doses were increased from 3 to $5-7 \mathrm{mg} / \mathrm{kg} /$ infusion. The higher dosage is given as a range to reflect the fact that, in our practice, infliximab as prescribed is routinely rounded upward to the nearest $100 \mathrm{mg}$ increment (ampoule size). Our dataset does not record the exactly calculated amount by actual body weight at the time of each infusion. At our institution during the period of this study, all infusions were given at eight week intervals throughout. Comparison of DAS28 values at the last evaluation before dose increase (that is, at the time when the decision to increase the dose was made) with the values after dose escalation showed a significant improvement at the first assessment following the dose increase 4.0 (0.18) months later (mean (SD); median 3.0), as shown in fig 1A. When we also compared the best DAS28 result seen in these patients at any time point after the dose increase, the improvement was maintained during further follow up. The best DAS28 results in this group of patients were achieved
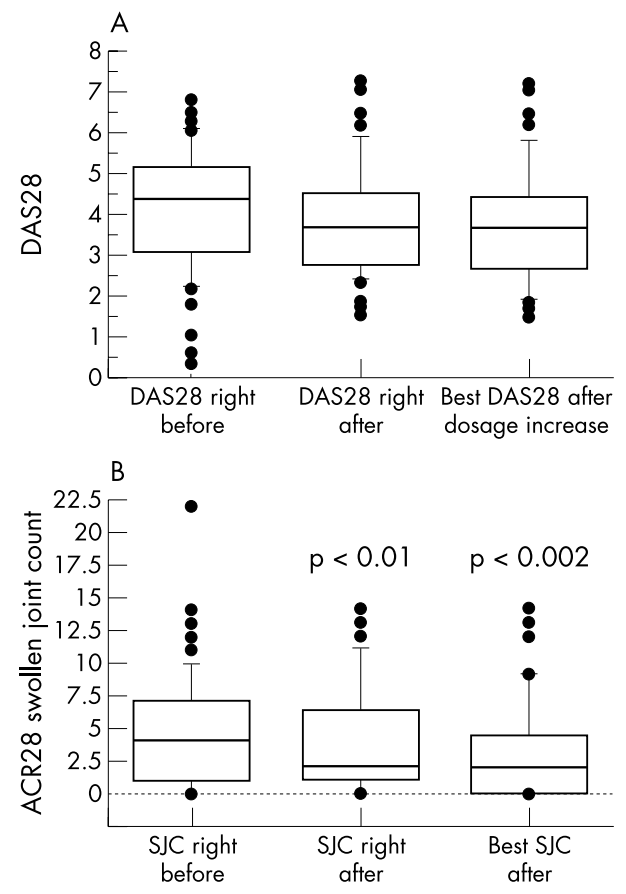

Figure 1 Infliximab dose escalation. DAS28 values (A) and 28 swollen joint counts (B) at the last visit before dose escalation, at the first visit following dose escalation, and the best result after dose escalation. Box plots show median values and $25-75 \%$ interquartile range. Statistical comparisons are by paired Student $t$ test. ACR28, American College of Rheumatology 28 joint score; DAS28, 28 joint count based disease activity score; SJC, swollen joint count.

after a mean of $7.2(0.56)$ months (range 3 to 18), and because infliximab infusions were always given at eight week intervals, this corresponded to a median of three infusions at the higher dose. The ACR28 swollen joint count following dose increase showed a significant improvement compared with the values at the last visit before the dose increase, and additional improvement thereafter (fig 1B).

For all outcomes, the quantitative degree of improvement was rather modest. For instance, the median improvement after dose increase in DAS28 was 0.6, which is just at the lower limit for the EULAR criterion of "moderate clinical responder." In an additional analysis, the clinical "response" to dose escalation was also analysed using established response criteria, and achieved a moderate to good EULAR response in 16 patients (36\%) and moderate to good ACR20 response in eight patients $(18 \%)$.

\section{Infliximab dose increases may recapture a previous response}

To assess the nature of the improvements seen with increasing doses, further comparisons were made by including each patient's best result at any time point before the dose increase. The patient's best DAS28 values at any time before the dose increases were significantly lower (that is, better) than the levels immediately preceding the dose increase $(3.46(0.17) \vee 4.04(0.19), \mathrm{p}<0.0001)$, and similar highly significant differences were seen for the other outcomes (not shown). This simply reflects the fact that the decision to increase the infliximab dose was made when disease activity had worsened. On average, the time from the best result on conventional dose infliximab until dose escalation was $3.7(0.53)$ months (range 3 to 15).

As seen in fig 2, the best DAS28 and best swollen joint count obtained after the dose increase-while being better than the results immediately before the dose increase-were 

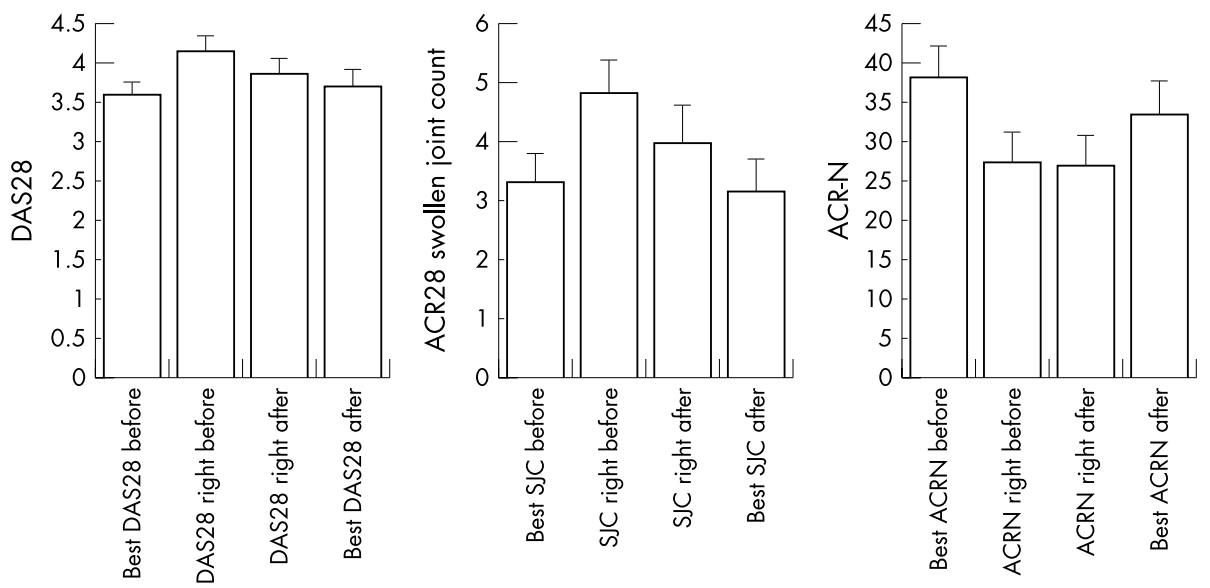

Figure 2 Comparisons of DAS28 (left panel), ACR28 swollen joint count (middle panel), and ACR-N (right panel) before and after dose escalation. Shown are, from left to right in each panel: the best value at any time point before the dose increase; the values at the time of the last visit before the dose increase; the values at the first visit after the dose increase; and the best values at any time point after dose increase. The results clearly show that the best result after dose escalation is similar to the best result before dose escalation. Error bars =SEM. ACR28, American College of Rheumatology 28 joint score; ACR-N, numeric ACR score; DAS28, 28 joint count based disease activity score; SJC, swollen joint count.

similar to the best results obtained at any previous time point before the dose increase. Comparison of ACR-N before and after the dose increase showed a similar pattern. One tempting interpretation of these findings is that the dose increase served to "recapture" a response to infliximab that had diminished with time. However, this pattern of response could also be explained by a regression-like effect.

Of these 44 patients, eight experienced an infusion reaction during at least one infusion. This is similar to the frequency in all infliximab treated patients in our unit. There did not appear to be an increase in such infusion reactions around the time of the dose increases, and none of these infusion reactions was regarded as severe or resulted in discontinuation of the treatment.

\section{Improvement after infliximab dose increase: comparison with controls}

To assess the observed improvement after infliximab dose escalation, we analysed two control groups-one group of patients treated with infliximab but in whom the dose was not increased, and one group treated with etanercept. As indicated above (Methods), the analysis of the control groups was based on the hypothesis that infliximab dose increases are "triggered" by worsening clinical results. We made the following comparisons: first, of the best values for DAS28, swollen joint count, and ACR-N at any time before the dose increase or the reference time point; second, of the values at the time of dose increase or the reference time point; and third, of the best values after the dose increase or the reference time point.

The time intervals between the best previous result and the reference time point in these control groups were $4.2(0.39)$ months (range 3 to 6 ) for the infliximab controls, and 4.5 (1.83) months (range 3 to 9) for the etanercept controls.

Because these three groups of patients were not randomised, we analysed whether changes in other drug treatments could have influenced our results. At the last visit before the dose increase or the reference time point, around half the patients in each group were taking daily oral glucocorticoids (prednisolone), at a mean dose of 5.4 (0.36) $\mathrm{mg}$ /day. At the first visit following that time point, the mean daily dose was $4.6(0.32) \mathrm{mg} /$ day — a small but statistically significant decrease. This was seen in all three groups, the changes in dose being similar and not statistically different in each group (not shown). However, these data had a nonnormal distribution, a few patients having large reductions to account for the overall reduced dosage. The median change in prednisolone dose for each group was zero. With respect to other DMARDs, most of the patients were taking concomitant methotrexate. There were no dose increases for either methotrexate or other DMARDs in conjunction with the infliximab dose escalations.

As can be seen in fig 3A, DAS28 values in the three groups showed very similar patterns. For the cases, the values at the last visit before the dose increase were worse than previous values, and after the dose increase they become better again ("recapturing the response"). However, in the controls, the pattern was similar - that is to say, the values at the reference time point were worse than previously (this following logically from the manner in which the reference time point was defined), but also showing an "improvement" after the reference time point, resulting in similar values to those obtained before. The time from reference time point to the best DAS28 result was $10.3(0.70)$ months (range 6 to 18 ) for the etanercept controls, and $7.2(0.56)$ months (range 0 to 18) for the infliximab controls, similar to the infliximab cases. Swollen joint counts and ACR-N values for this analysis are shown in fig $3 \mathrm{~B}$ and $3 \mathrm{C}$, and reveal similar patterns, but with the difference that in the "cases" the ACR$\mathrm{N}$ responses at each time point were lower than in the controls, while they showed the same pattern of worsening followed by improvement. Thus the changes in outcome in the three groups were numerically similar and not statistically different.

\section{DISCUSSION}

We used a structured longitudinal follow up registry of patients treated with biological agents to study the effects in clinical practice of increasing the dose of infliximab above $3 \mathrm{mg} / \mathrm{kg} /$ infusion. Such dose increases occur quite often and are usually occasioned by insufficient clinical response, although insufficient duration of response (that is, less than eight weeks) is also commonly given as the reason. This may be surprising in that it makes intuitive sense to increase the frequency rather than the dose in such an event. However, until 2001 no data were available to indicate which course of action would be best, and increasing the frequency of infusions was discouraged owing to the limited availability 

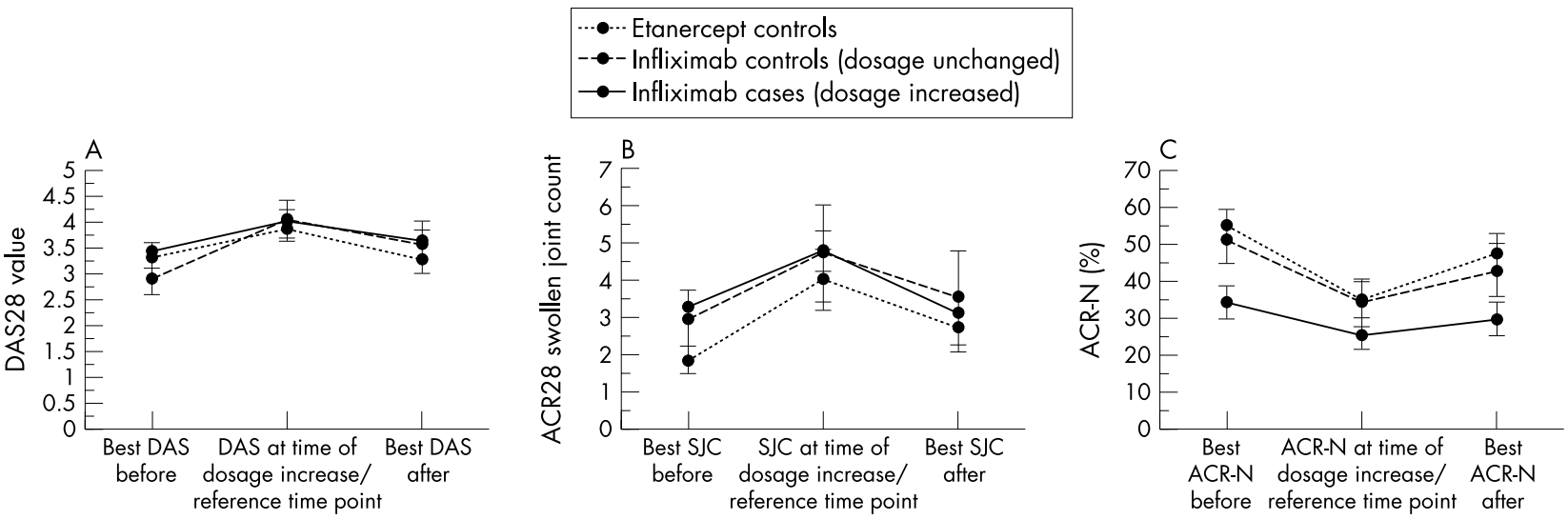

Figure 3 Case-control study: graphs showing DAS28 (A), ACR28 swollen joint counts (B), and ACR-N (C) for cases (patients whose infliximab dosages were increased: solid lines), infliximab controls (whose dosages were not increased: dashed lines), and etanercept controls (dotted lines). The values plotted are the best results before dose escalation (for the cases) or before the reference time point (for the controls); the values at the last visit before these time points; and the best values thereafter. Error bars =SEM. ACR28, American College of Rheumatology 28 joint score; ACR-N, numeric ACR score; DAS28, 28 joint count based disease activity score; SJC, swollen joint count.

of nursing resources for intravenous treatments. A recently published pharmacokinetic modelling study ${ }^{9}$ suggested that increasing the frequency of infusions was more effective for such patients than increasing the dose, and this is likely to affect these types of decisions in the future.

Thus for most patients the dose increase was prompted by an insufficient clinical response. Our analysis shows that following dose increases, better results were seen, such that the average DAS28, swollen joint count, and ACR-N improved and $18-36 \%$ of patients met formal response criteria. On the other hand, the improvements were rather modest in magnitude, and the values after dose escalation were similar to previously achieved best results; they might therefore be interpreted as showing that a previous response to infliximab treatment that had diminished over time could be "recaptured" by the dose increase. However, in our casecontrol analysis, we were led to conclude that a more frugal explanation for these findings is a regression-like effectthat is, inasmuch as dose increases are prescribed at a time when the patients are doing relatively poorly, it is more than likely that they will do better again at time points thereafter. Thus in the control groups we chose a reference time point to mirror the time at which a dose increase could have been prescribed-namely, those times at which a worsening of clinical outcomes had occurred. The two control groups showed a similar pattern of worsening and "recapture" to the cases. The time to achieve the best result was similar for the three groups (the mean for all patients was 8.6 months, with a range from 3 to 18 months), which would appear long enough to allow maximum responses to dose escalation to occur. Of note, the ACR-N values were overall somewhat worse for the cases, suggesting that this is a patient group with a less favourable clinical course, which may indeed be the reason why the doses were in fact increased in these patients and not in the infliximab controls.

There were no statistically significant differences between the changes in the outcome measures for the cases and the controls at any of the time points. However, it could be argued that a small but true difference might be missed in this analysis of just over 100 patients (type 2 error). We would counter that any true difference in outcomes that went unnoticed in this study would have to be of such small magnitude that its practical relevance would be extremely limited.

The improvements seen in our patients following a period of worsening disease activity, irrespective of treatment, is typical of the waxing and waning course of rheumatoid arthritis and many other chronic diseases. The appearance of an improvement when a subset of patients is analysed, starting at a time of worsened disease, causes an error in interpretation known in statistics as "regression to the mean." It has been suggested that the sometimes impressive responses seen in the placebo groups in clinical trials are explained to a much greater extent by regression to the mean than by the actual placebo effect. ${ }^{10} 11$

One could speculate that infliximab dosage increases might be prompted by decreasing efficacy as a result of antiinfliximab antibody formation. We cannot at this time test for such antibodies. However, the lack of a measurable increase in infusion reactions in these patients around the time of the dose increase would argue indirectly against this possibility.

The cost of infliximab is very high, and the typical dose increase doubles the cost of the treatment. In addition, some risks may be greater with higher doses. As indicated above, in patients with severe pre-existing congestive heart failure, increased mortality was seen with the higher infliximab dosage. $^{2}$ In the ATTRACT clinical trial, while the total number of malignancies was not higher than expected, the distribution of malignancies within the treatment groups was skewed towards the higher dosages. ${ }^{12}$ And in previous studies with another TNF $\alpha$ blocking agent, D2E7 (adalimumab, Humira), a risk for tuberculosis was observed, especially at high dosages. ${ }^{13}$ Thus a burden is imposed on physicians who elect to prescribe higher infliximab dosages to assess the results of this intervention. When informally reviewing the cases in this study we found, rather soberingly, that the treating physicians tended to assess the results as more favourable than was warranted by the actual outcomes, and that even when the results were assessed as being no better than before, the dose increases were rarely reversed.

We conclude that dosage increases of infliximab may have less clinical benefit than is expected or perceived in clinical practice, and that much of the perceived benefit is, in fact, a regression effect. We recommend that formal studies of infliximab dose escalation be undertaken and that individual physicians wishing to increase infliximab dosage do so under very clearly prespecified conditions of follow up. We also conclude that use of formal surveillance systems similar to the one used in this study should be encouraged when introducing new drugs, in order to permit optimisation of their use in clinical practice and to avoid 
substantial costs for perceived but scientifically unverifiable patient benefits.

\section{Authors' affiliations}

R F van Vollenhoven, S Brannemark, L Klareskog, Department of Rheumatology, Karolinska Hospital, 17176 Stockholm, Sweden

\section{REFERENCES}

1 Lipsky PE, van der Heijde DM, St Clair EW, Furst DE, Breedveld FC, Kalden JR, et al. Infliximab and methotrexate in the treatment of rheumatoid arthritis. Anti-Tumor Necrosis Factor Trial in Rheumatoid Arthritis with Concomitant Therapy Study Group. N Engl J Med 2000;343:1594-602.

2 Coletta AP, Clark AL, Banarjee P, Cleland JG. Clinical trials update: RENEWAL (RENAISSANCE and RECOVER) and ATTACH. Eur J Heart Fail 2002:4:559-6.

3 Pincus T. Limitations of randomized controlled clinical trials to depict accurately long-term outcomes in rheumatoid arthritis. $Z$ Rheumatol 1998;57:46-9.

4 Van der Heiide DMFM, van 't Hof MA, van Riel PLCM, van de Putte LBA. Development of a disease activity score based on judgement in clinical practice by rheumatologists. J Rheumatol 1993;20:579-81.

5 Prevoo MLL, van ' $t$ Hof MA, Kuper HH, van Leeuwen MA, van de Putte $L B$, van Riel PL. Modified disease activity scores that include twenty-eight-joint counts. Arthritis Rheum 1995:38:44-8.
6 Bathon JM, Martin RW, Fleischmann RM, Tesser JR, Schiff MH, Keystone EC, et al. A comparison of etanercept and methotrexate in patients with early rheumatoid arthritis. N Engl J Med 2000;343:1586-93.

7 van Gestel AM, Prevoo MLL, van ' $\nmid$ Hof MA, van Rijswijk MH, van de Putte LB, van Riel PL. Development and validation of the European League Against Rheumatism response criteria for rheumatoid arthritis. Arthritis Rheum 1996;39:34-40.

8 Felson DT, Anderson JJ, Boers M, Bombardier C, Furst D, Goldsmith C, et al. The American College of Rheumatology preliminary definition of improvement in rheumatoid arthritis. Arthritis Rheum 1995;38:727-35.

9 St Clair EW, Wagner CL, Fasanmade AA, Wang B, Schaible T, Kavanaugh A, et al. The relationship of serum infliximab concentrations to clinical improvement in rheumatoid arthritis: results from ATRACT, a multicenter, randomized, double-blind, placebo-controlled trial. Arthritis Rheum 2002;46:1451-9.

10 Senn SJ. How much of the placebo "effect" is really statistical regression? Stat Med 1988;7:1203.

11 Hrobjartsson A, Gotzsche PC. Is the placebo powerless? An analysis of clinical trials comparing placebo with no treatment. N Engl I Med 2001;344:1594-602

12 Maini R, St Clair EW, Breedveld FC, Furst D, Kalden J, Weisman M, et al. Infliximab (chimeric anti-tumour necrosis factor $\alpha$ monoclonal antibody) versus placebo in rheumatoid arthritis patients receiving concomitant methotrexate: a randomised phase III trial. Lancet 1999;354:1932-9.

13 Kempeni J. Preliminary results of early clinical trials with the fully human antiTNFalpha monoclonal antibody D2E7. Ann Rheum Dis 1999;58(suppl 1):170-2. 